\title{
تقويم بيانات العمر والنوع للتعداد العام للسكان في العراق باستعمال هقدرات Kernel Bayesian اللاهملمية
}

\author{
أ.م.د. قتيبة نبيل نايف القزاز الباحثة/ مروة خليل ابراهيم

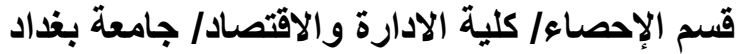

تعد عملية تقويم بيانات التركيب العمري والنوعي من العوامل المهمة التي تساعد اي دولة التئة في رسم

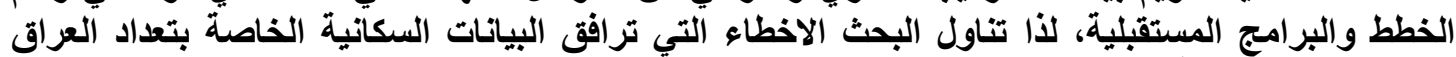

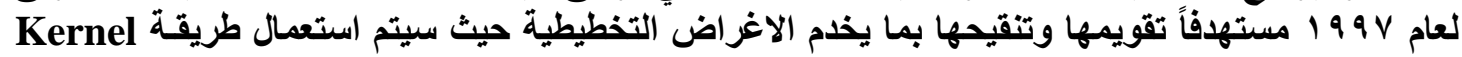
اللامعلميـة بواسطة مقدر (Nadaraya-Watson) لايجاد معادلة الانحدار التقديرية لتمهيد البيانات

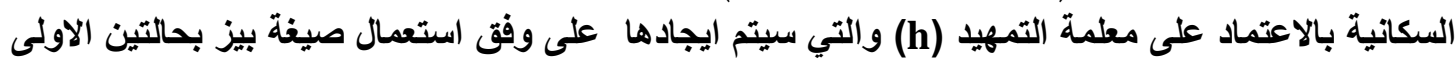

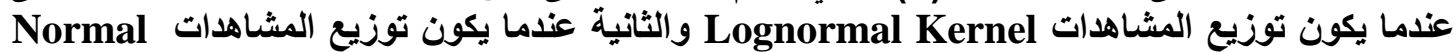
Kernel

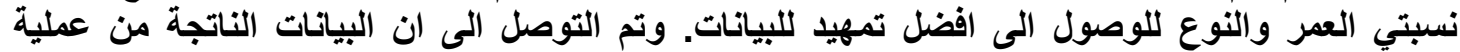
التمهيخ بطريقة تقدير Kernel وحسب طريقة ايجاد معلمة التمهيد

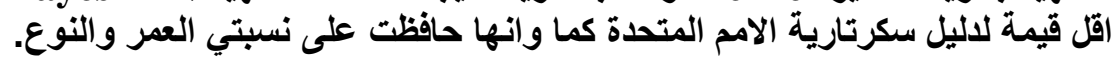

الإسطاحات الرئيسية لابحث/ مقدرات Nadaraya-Watson Kernel- توزيع مقلوب كاما السابق- توزيع Lognormal Kernel اللاحق- توزيع Normal Kernel اللاحق- مقياس الامم المتحدة 
ان لتقويم وتصحيح البيانات السكانية اهمية كبيرة للحصول على بيانات خالية من الاخطاء واستعمالها

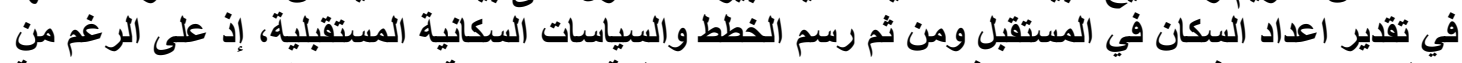

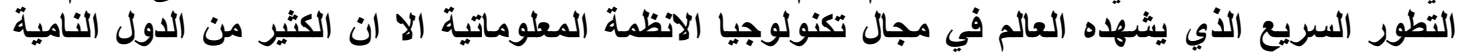

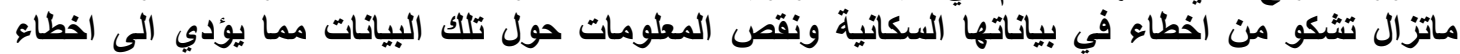

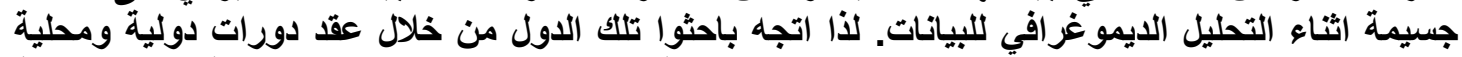

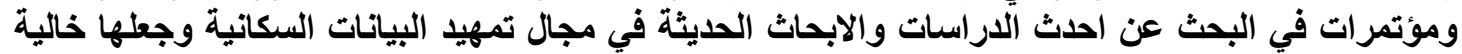
ويقلر مقبول من الاخطاء.

تتعرض بيانات التركيب العمري و النوعي لعدد من الاخطاء يمكن تقسيمها على قسمين: [2],[3],]

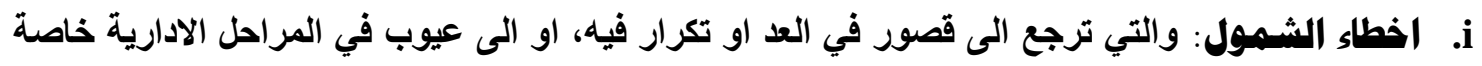

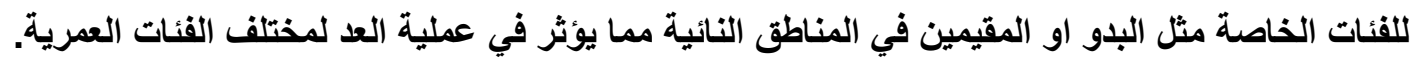
ii

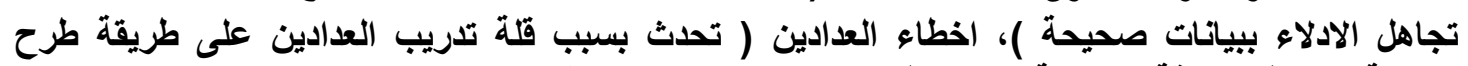

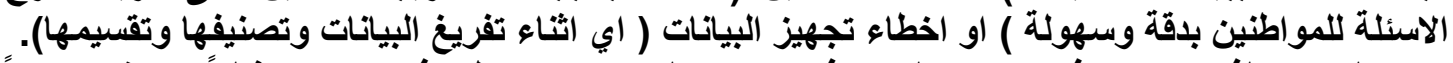

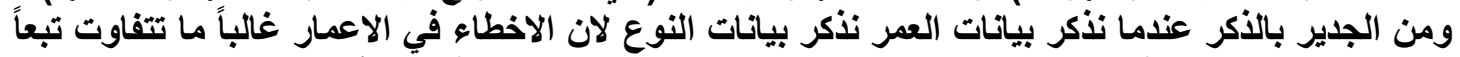

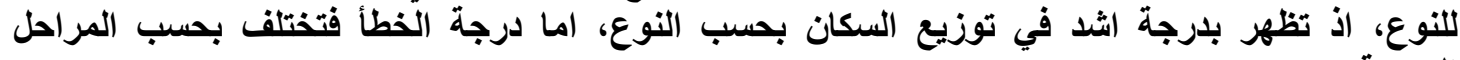

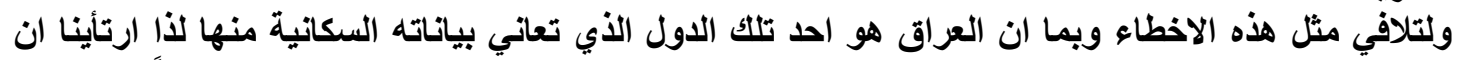

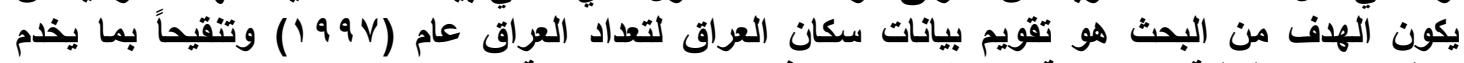

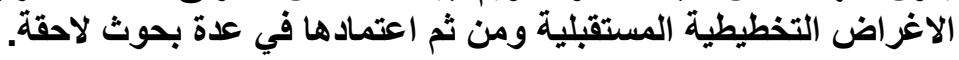

\section{r. طرائق تمهيد بيانات التعداد: [6],[11]}

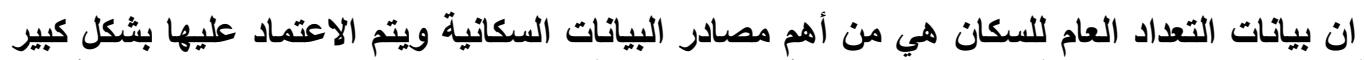

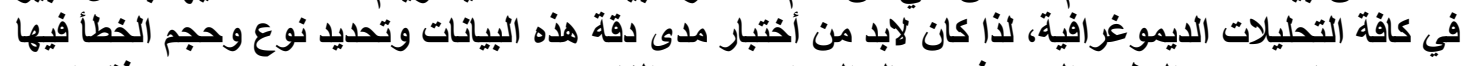

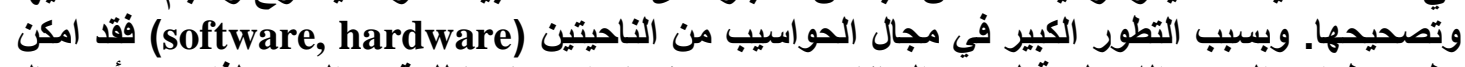

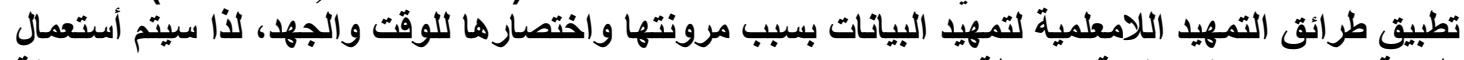

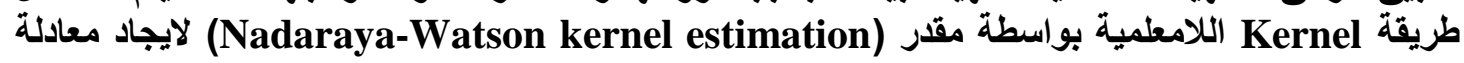

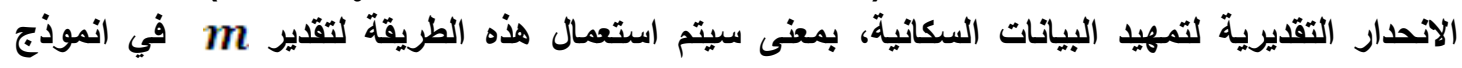

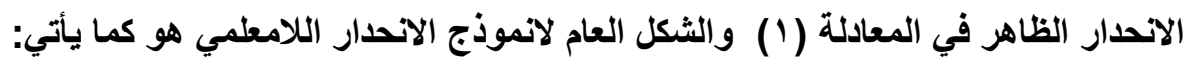

$$
Y_{i}=m\left(X_{i}\right)+\epsilon_{i} \quad i=1, \ldots, n
$$


وان طريقة Nadaraya-Watson kernel estimation أُقترحت بواسطة Nadaraya و $\widehat{m}_{h}(x)=\frac{\sum_{i=1}^{n} K_{h}\left(x-X_{i}\right) y_{i}}{\sum_{i=1}^{n} K_{h}\left(x-X_{i}\right)}$ في عام (ـ 9 (1) ) وسُميت على اسمهما.

$\because W_{h}\left(x-X_{i}\right)=\frac{K_{h}\left(x-X_{i}\right)}{\sum_{i=1}^{n} K_{h}\left(x-X_{i}\right)}$

$\therefore \widehat{m}_{h}(x)=\sum_{i=1}^{n} W_{h}\left(x-X_{i}\right) y_{i}$

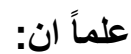

Kernel تمثل دالة $K_{h}\left(x-X_{i}\right)$

h تمثل عرض الحزمسة (bandwidth) او تعرف بمعلمة التمهيا (smooth parameter)، وهي عبـارة

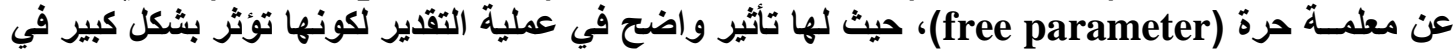

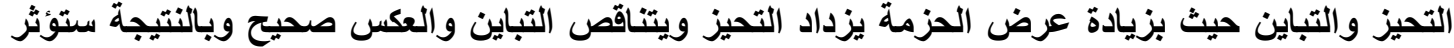
$\sum_{i=1}^{n} W_{h}\left(x-X_{i}\right)=1$ في تمهيد المنحنى ومعدل اقترابه من المنحنى الحقيقي.

وفي اي انحدار لامعلمي التوقع الثرطي للمتغير X X بالنسبة الى المتغير X بالثكل الاتي: $E(Y \mid X)=m(X)$

$$
E(Y \mid X)=\int y f(y \mid x) d y=\int y \frac{f(x, y)}{f(x)} d y
$$

ولاشتقاق الصيغة في المعادلة (T)

سنستعمل مقدر كثافة Kernel للتوزيع المشترك (x,y)

$\hat{f}(x, y)=n^{-1} h^{-2} \sum_{i=1}^{n} K\left(\frac{x-X_{i}}{h}\right) K\left(\frac{y-y_{i}}{h}\right)$

$$
=\frac{1}{n} \sum_{i=1}^{n} K_{h_{x}}\left(x-X_{i}\right) K_{h_{y}}\left(y-y_{i}\right)
$$

$\int y \hat{f}(x, y) d y=\frac{1}{n} \int y \sum_{i=1}^{n} K_{h_{x}}\left(x-X_{i}\right) K_{h_{y}}\left(y-y_{i}\right)$

$\because \int y K_{h_{y}}\left(y-y_{i}\right) d y=y_{i}$ 


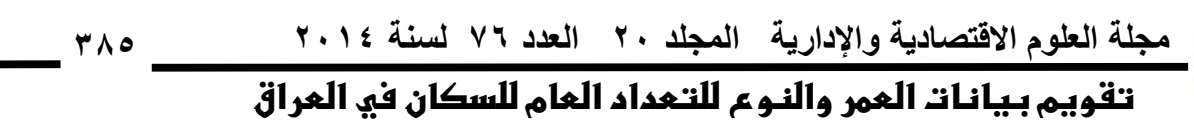

باستعمال مقدرات Kernel Bayesian اللامعلمية

$\therefore \int y \hat{f}(x, y) d y=\frac{1}{n} \sum_{i=1}^{n} K_{h_{x}}\left(x-X_{i}\right) y_{i}$

ان المعادلة (8) تمثل مقدر البسط للمعادلة (2).

$\int \hat{f}(x, y) d y=\frac{1}{n} \sum_{i=1}^{n} K_{h_{x}}\left(x-X_{i}\right) \int K_{h_{y}}\left(y-y_{i}\right) d y$

$=\frac{1}{n} \sum_{i=1}^{n} K_{h_{x}}\left(x-X_{i}\right)$

$=\frac{1}{n h} \sum_{i=1}^{n} K\left(\frac{x-X_{i}}{h}\right)$

$\hat{f} \hat{f}(x)$

اما المعادلة (9) فتمثل مقدر المقام للمعادلة (2)، ومن المعادلتين (8) و (9) سنحصل على مقار

Nadaraya-Watson Kernel

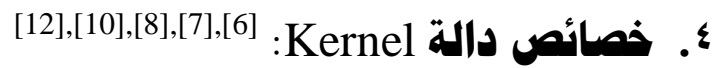
، $K(u) \geq 0$ الكل كثافة احتماليـة محدة (غير سالبة) بحيث

$M_{j}(K)=\int_{-\infty}^{\infty} u^{j} K(u) d(u)$

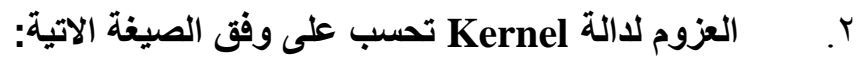

3. $\int_{-\infty}^{\infty} u K(u) d(u)=0$

4. $C_{K}=R(K)=\int_{-\infty}^{\infty} K^{2}(u) d(u)$

5. $\quad d_{K}=M_{2}(K)=\int_{-\infty}^{\infty} u^{2} K(u) d(u)$

ومن الجدير بالملاحظة ان القيمة

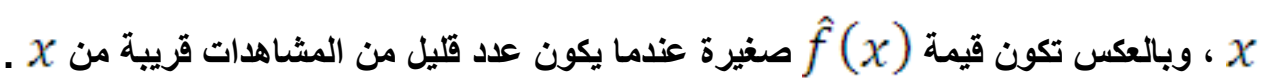

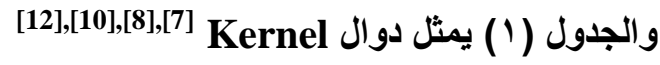


باستعمال مقدرات Kernel Bayesian اللامعلمية

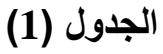

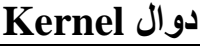

\begin{tabular}{|l|c|c|}
\hline \multicolumn{1}{|c|}{ Kernel } & \multicolumn{2}{|c|}{$K(\boldsymbol{u})$} \\
\hline Uniform (p=0) & $1 / 2$ &, $\mathrm{I}(|u| \leq 1)$ \\
\hline Epanchnikov (p=1) & $\frac{3}{4}\left(1-u^{2}\right)$ &, $\mathrm{I}(|u| \leq 1)$ \\
\hline $\left.\begin{array}{l}\text { Quartic } \\
\text { or } \\
\text { Biweight }\end{array}\right\}(\mathbf{p = 2})$ & $\frac{15}{16}\left(1-u^{2}\right)^{2}$ &, $\mathrm{I}(|u| \leq 1)$ \\
\hline $\begin{array}{l}\text { Triwight }(\mathbf{p}=\mathbf{3}) \\
\text { Gauss }\end{array}$ & $\frac{35}{32}\left(1-u^{2}\right)^{3}$ &, $\mathrm{I}(|u| \leq 1)$ \\
\hline
\end{tabular}

و هناك طرائق عدة لايجاد عرض الحزمة منها استعمال اسلوب بيز.

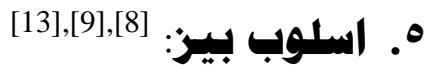

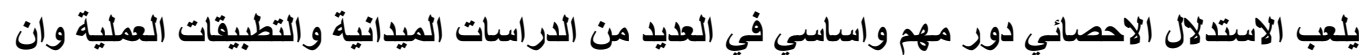

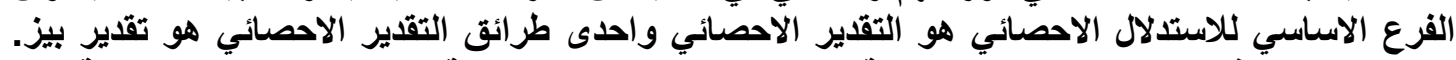

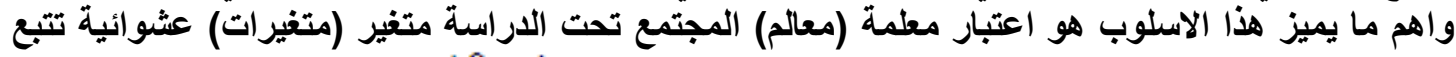

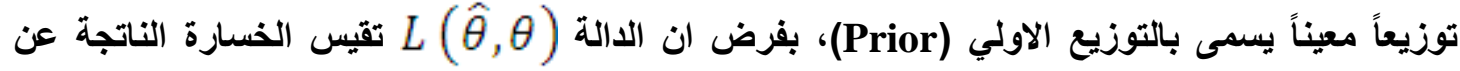
اختيار الاجراء الخسارة المناسبة يكون الهدف هو اختيار المقدر الذي يجعل مخاطرة بيز Bayes risk اقل مايمكن عندما نقلر المعلمة $\theta$ بالمقدرف، اي ان مقدر بيز بالنسبة للتوزيع اللاحق اقل مايمكن.

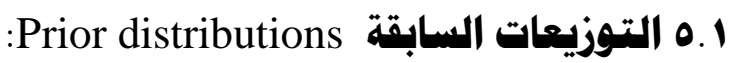

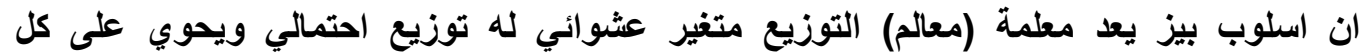

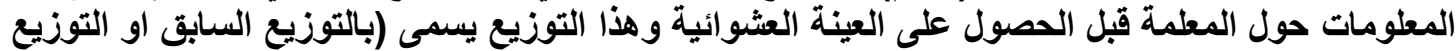

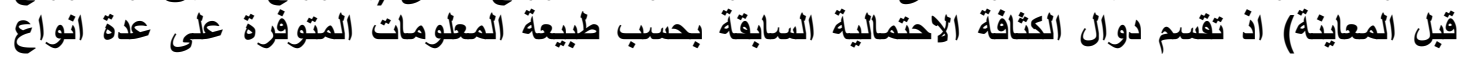
منها:

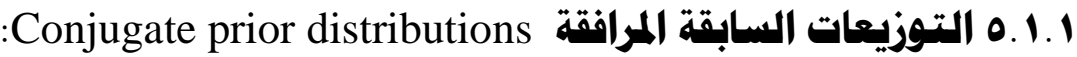

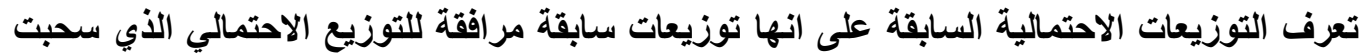

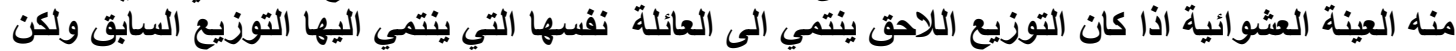
بمعلمات مختلفة. 
باستعمال مقدرات Kernel Bayesian الإمعلمية

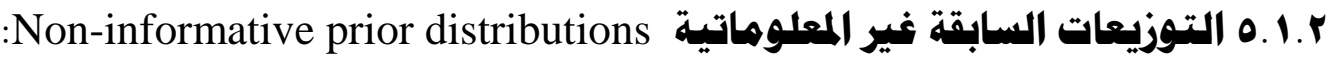
وهي عبارة عن توزيعات احتمالية تعبر عن المعرفة القليلة او شبه المعلومة لمعلمة (معالم) التوزيع

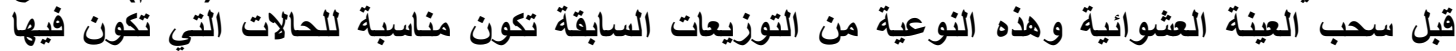

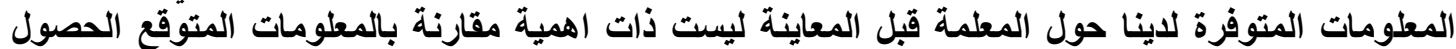
عليها من العينة العثوائية.

:Posterior distributions

ب.r التوزيعات اللاحقة

وهي التي تصف درجة الاعتقاد حول القيم الممكنة للمعلمة بعد الحصول على العينة، ويسمى التوزيع اللاحق بالتوزيع بعد المعاينة. ويرمز له بالرمز (ب|x

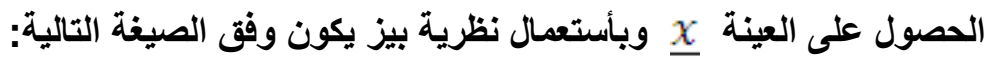
$\pi^{*}(\theta \mid \underline{x})=\frac{\pi(\theta) l(\theta \mid \underline{x})}{\int_{\theta} \pi(\theta) l(\theta \mid \underline{x}) d \theta}$ حيث (i)

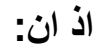

$\pi^{*}(\theta \mid \underline{x}) \propto \pi(\theta) l(\theta \mid \underline{x})$

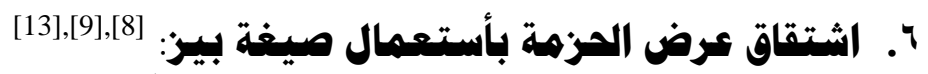

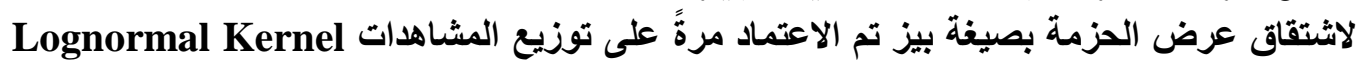

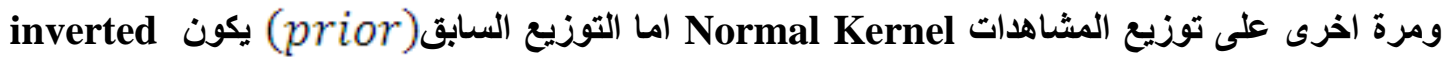

gamma

:Lognormal Kernel توزيع المشاهدات: a

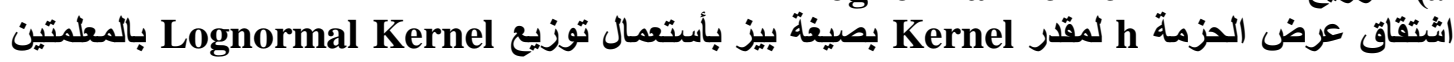
$\mu, \sigma$

$K(x, \mu, \sigma)=\frac{1}{\sqrt{2 \pi \sigma^{2}} x} e^{-\frac{1}{2}\left(\frac{\ln x-\mu}{\sigma}\right)^{2}}=\frac{1}{x h \sqrt{2 \pi}} e^{-\frac{1}{2}\left(\frac{\ln x-\ln z_{j}}{h}\right)^{2}}$

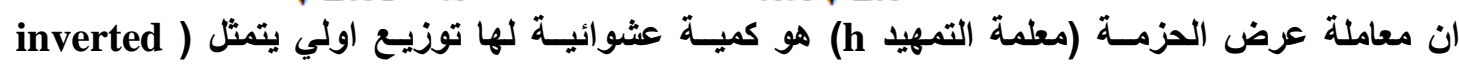
$\pi(h)=\frac{1}{\beta^{\alpha} \Gamma \alpha h^{\alpha+1}} e^{-\frac{1}{\beta h}} \quad, h>0$

$\hat{f}_{h}(x)=\sum_{i=1}^{n} K\left(x, \ln z_{j}, h\right)$

حيث: 


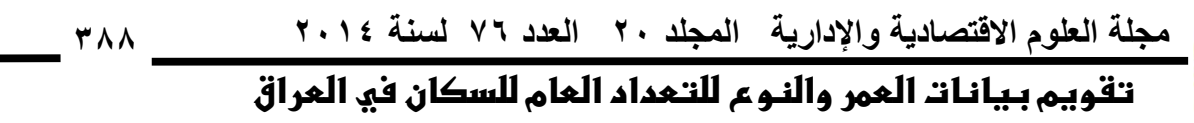

باستعمال مقدرات Kernel Bayesian اللامعلمية

دالة الكثافة الاحتمالية اللاحقة لا (h) تكون:

$\pi^{*}(h \mid x, \underline{z})=\frac{f_{h}(x) \pi(h)}{\int f_{h}(x) \pi(h) d h}$

ويما ان $f_{h}$ غير معلومة، تم استعمال

$\hat{\pi}^{*}(h \mid x, \underline{z})=\frac{\hat{f}_{h}(x) \pi(h)}{\int \hat{f}_{h}(x) \pi(h) d h}$

$\int \hat{f}_{h}(x) \pi(h) d h=\int_{0}^{\infty} \sum_{j=1}^{n} \frac{1}{x h \sqrt{2 \pi}} e^{-\frac{1}{2}\left(\frac{\ln x-\ln z_{j}}{h}\right)^{2}} \cdot \frac{1}{\beta^{\alpha} \Gamma \alpha h^{\alpha+1}} e^{-\frac{1}{\beta h}} d h$

نجد هنالك اختلاف بين معلمة التوزيع السابق ومعلمة توزيع Lognormal Kernel وبأستعمال

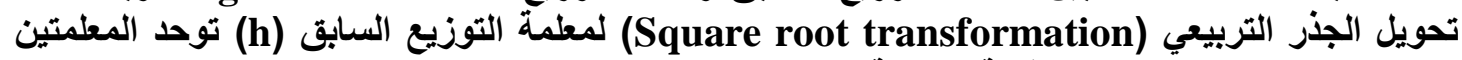

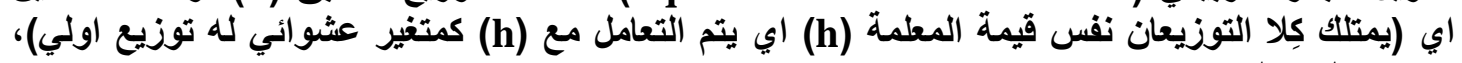

$$
\begin{aligned}
f_{Y}(y) & =f_{X}\left(g^{-1}(y)\right) \cdot\left|\frac{d}{d y} g^{-1}(y)\right| \\
& =f_{X}\left(y^{2}\right) \cdot 2 y \\
& =\frac{1}{\beta^{\alpha} \Gamma \alpha\left(y^{2}\right)^{\alpha+1}} \cdot e^{-\frac{1}{\beta y^{2}}} \cdot 2 y \\
& =\frac{1}{\beta^{\alpha} \Gamma \alpha y^{2 \alpha+1}} \cdot e^{-\frac{1}{\beta y^{2}}} \cdot 2
\end{aligned}
$$

ولايجاد التحويل:

اذا كانت $\pi(h)=\frac{2}{\beta^{\alpha} \Gamma h^{2 \alpha+1}} \cdot e^{-\frac{1}{\beta h^{2}}} \quad, h>0$ 


$$
\begin{aligned}
\int \hat{f}_{h}(x) \pi(h) d h & =\int_{0}^{\infty} \sum_{j=1}^{n} \frac{1}{x h} \frac{1}{\sqrt{2 \pi}} e^{-\frac{1}{2}\left(\frac{\ln x-\ln z_{j}}{h}\right)^{2}} \cdot \frac{2}{\beta^{\alpha} \Gamma \alpha h^{2 \alpha+1}} e^{-\frac{1}{\beta h^{2}}} d h \\
& =\int_{0}^{\infty} \sum_{j=1}^{n} \frac{1}{x} \frac{1}{\sqrt{2 \pi}} \frac{2}{\beta^{\alpha} \Gamma \alpha\left(h^{2}\right) \alpha+1} e^{-\frac{1}{h^{2}}\left(\frac{1}{\beta}+\frac{1}{2}\left(\ln x-\ln z_{j}\right)^{2}\right)} d h
\end{aligned}
$$

$$
\begin{aligned}
& \beta_{\mathrm{j}}^{*}=\left[\frac{1}{\beta}+\frac{1}{2}\left(\ln x-\ln Z_{j}\right)^{2}\right]^{-1} \\
& \alpha^{*}=\alpha+\frac{1}{2}
\end{aligned}
$$

$$
\begin{aligned}
\int \hat{f}_{h}(x) \pi(h) d h & =\int_{0}^{\infty} \sum_{j=1}^{n} \frac{1}{\sqrt{2 \pi}} \frac{1}{x} e^{-\frac{1}{t \beta_{j}^{8}}} \frac{1}{\beta^{\alpha} \Gamma \alpha t^{\alpha+1}} \frac{1}{\sqrt{t}} d t \\
& =\frac{1}{x} \sum_{j=1}^{n} \frac{\left(\beta_{j}^{*}\right)^{\alpha^{8}} \Gamma\left(\alpha^{*}\right)}{\sqrt{2 \pi} \beta^{\alpha} \Gamma \alpha} \int_{0}^{\infty} \frac{1}{\left(\beta_{j}^{*}\right)^{\alpha^{8}} \Gamma\left(\alpha^{*}\right) t^{\alpha^{8}+1}} e^{-\frac{1}{t \beta_{j}^{8}} d t}
\end{aligned}
$$

وبذلك يكون المقام في المعادلة (20):

$$
\begin{aligned}
& \frac{\Gamma\left(\alpha^{*}\right)}{x \Gamma \alpha} \sum_{j=1}^{n} \frac{\left(\beta_{j}^{*}\right)^{\alpha^{*}}}{\sqrt{2 \pi} \beta^{\alpha}} \\
& \hat{f}_{h}(x) \pi(h)=\sum_{j=1}^{n} \frac{1}{\sqrt{2 \pi}} \frac{1}{x} \frac{2}{\beta^{\alpha} \Gamma \alpha\left(h^{2}\right)^{\alpha+1}} e^{-\frac{1}{h^{2} \beta_{j}^{8}}}
\end{aligned}
$$

وبالتالي، فأن مقدر الكثافة الاحتمالية اللاحقة لا h هو:

$$
\widehat{\pi}(h \mid x, \underline{z})=\frac{\sum_{j=1}^{n} \frac{1}{\sqrt{2 \pi}} \frac{1}{x} \frac{2}{\beta^{\alpha} \Gamma \alpha\left(h^{2}\right) \alpha+1} e^{-\frac{1}{h^{2} \beta_{j}^{8}}}}{\frac{\Gamma\left(\alpha^{8}\right)}{\Gamma \alpha(x)} \sum_{j=1}^{n} \frac{\left(\beta_{j}^{8}\right)^{\alpha^{8}}}{\sqrt{2 \pi} \beta^{\alpha}}}
$$

$$
=\frac{\sum_{j=1}^{n} \frac{2}{\left(h^{2}\right)^{\alpha+1}} e^{-\frac{1}{h^{2} \beta_{j}^{8}}}}{\Gamma\left(\alpha^{*}\right) \sum_{j=1}^{n}\left(\beta_{j}^{*}\right)^{\alpha^{8}}}
$$

وتحت دالة الخسارة التربيعية فأن مقدر بيز لمعلمة h (والأي يمثل متوسط التوزيع اللاحق) يكون: 


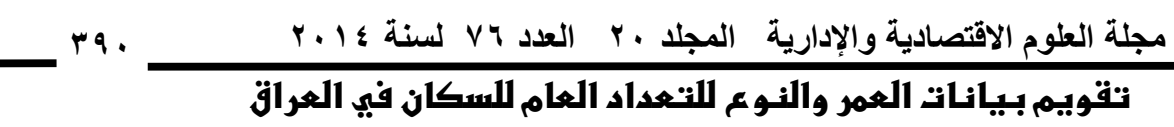

باستعمال مقدرات Kernel Bayesian اللامعلمية

$$
\begin{aligned}
\tilde{h}(x) & =\int_{0}^{\infty} h \hat{\pi}(h \mid x, \underline{z}) d h \\
& =\int_{0}^{\infty} h \frac{\sum_{j=1}^{n} \frac{2}{\left(h^{2}\right)^{\alpha+1}} e^{-\frac{1}{h^{2} \beta_{j}^{\beta}}}}{\Gamma\left(\alpha^{*}\right) \sum_{j=1}^{n}\left(\beta_{j}^{*}\right)^{\alpha^{*}}} d h \\
& =\frac{1}{\Gamma\left(\alpha^{*}\right) \sum_{j=1}^{n}\left(\beta_{j}^{*}\right)^{\alpha^{8}}} \int_{0}^{\infty} h \sum_{j=1}^{n} \frac{2}{\left(h^{2}\right)^{\alpha+1}} e^{-\frac{1}{h^{2} \beta_{j}^{*}}} d h
\end{aligned}
$$

وبأستبدال

$\tilde{h}(x)=\frac{1}{\Gamma\left(\alpha^{*}\right) \sum_{j=1}^{n}\left(\beta_{j}^{*}\right)^{\alpha^{*}}} \sum_{j=1}^{n} \int_{0}^{\infty} \frac{1}{(t)^{\alpha+1}} e^{-\frac{1}{t \beta_{j}^{*}}} d t$

يمكنتا تبسيط التكامل في المعادلة (32) كالاتي:

$\tilde{h}(x)=\frac{\Gamma \alpha \sum_{j=1}^{n}\left(\beta_{j}^{*}\right)^{\alpha^{8}}}{\Gamma\left(\alpha^{*}\right) \sum_{j=1}^{n}\left(\beta_{j}^{*}\right)^{\alpha^{8}}} \int_{0}^{\infty} \frac{1}{\left(\beta_{j}^{*}\right)^{\alpha^{*}} \Gamma \alpha(t)^{\alpha+1}} e^{-\frac{1}{t \beta_{j}^{8}}} d t$

$\tilde{h}(x)=\frac{\Gamma \alpha \sum_{j=1}^{n}\left(\beta_{j}^{*}\right)^{\alpha^{8}}}{\Gamma\left(\alpha^{*}\right) \sum_{j=1}^{n}\left(\beta_{j}^{*}\right)^{\alpha^{8}}}$

يكون عرض الحزمة h بصيغة بيز هو:

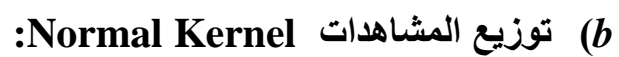
وبنفس الطريقة السابقة لايجاد عرض الحزمة الامثل h بتوزيع المشاهدات (Lognormal Kernel) تم

$$
\begin{aligned}
& K(u)=\frac{1}{\sqrt{2 \pi}} e^{\frac{-u^{2}}{2}}, \quad-\infty<u<\infty
\end{aligned}
$$

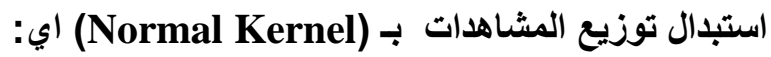

$$
\begin{aligned}
& u=\frac{x-X_{j}}{h}
\end{aligned}
$$$$
\pi(h)=\frac{2}{\Gamma \alpha \beta^{\alpha} h^{2 \alpha+1}} e^{-\frac{1}{\beta h^{2}}} \quad, \alpha>0
$$$$
\beta>0
$$$$
, h>0
$$

التوزيع السابق كما ذكر بالمعادلة (r Y ) وبعد استعمال التحويل لها فأن. 


$$
\hat{\pi}(h \mid x, \underline{z})=\frac{\sum_{j=1}^{n}\left(1 / h^{2 \alpha+2}\right) e^{-\frac{1}{h^{2}}\left(\frac{1}{\beta}+\frac{1}{2}\left(x-X_{j}\right)^{2}\right)}}{(\Gamma((\alpha+1) / 2) / 2) \sum_{j=1}^{n}\left(\frac{1}{\beta}+\frac{1}{2}\left(x-X_{j}\right)^{2}\right)}
$$

وبالتالي عرض الحزمة h لتوزيع المشاهدات (Normal Kernel) بصيغة بيز تكون:

$$
h^{*}(x)=\frac{\Gamma \alpha \sum_{j=1}^{n}\left(\frac{1}{\beta\left(x-X_{j}\right)^{2}}+2\right)^{\alpha}}{\sqrt{2 \beta} \Gamma\left(\alpha+\frac{1}{2}\right) \sum_{j=1}^{n}\left(1 /\left(\beta\left(x-X_{j}\right)^{2}+2\right)\right)^{\left(\alpha+\frac{1}{2}\right)}}
$$

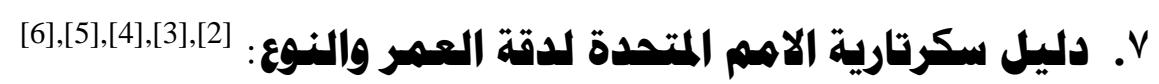

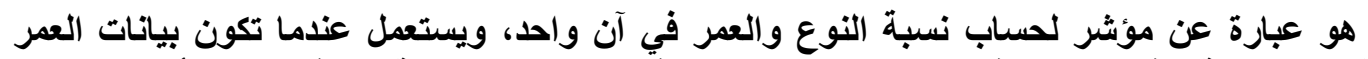

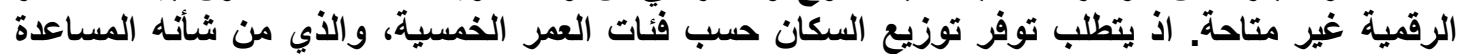

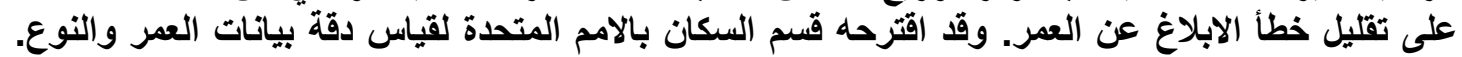

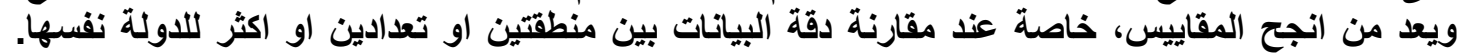

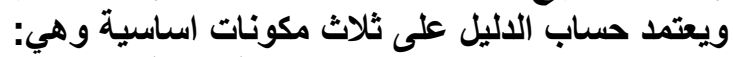

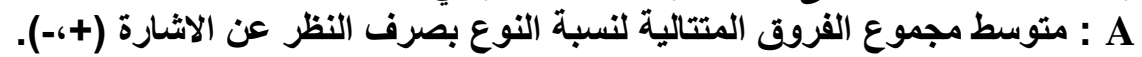
: B

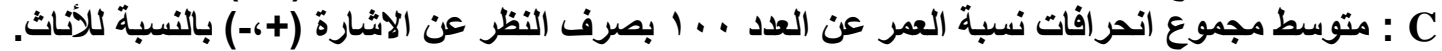
ويجري حساب قيمة الاليل وفق الصيغة الرياضية العية التالية: $U N_{M}=3 A+B+C$

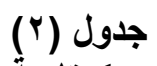
وتفسر نتيجة الاليل على النحو التالي:

\begin{tabular}{|c|c|}
\hline مستوى جودة البيانات & قيمة الدليل \\
\hline البيانات دقيقة ويمكن الاعتماد عليها & آقل من · r \\
\hline البيانات تعاني من ضعف بلرجة متوسطة & من · r الى • \\
\hline البيانات غير صحيحة وتشويها اخطاء كثيرة & اكثر من · ؛ \\
\hline
\end{tabular}

مستوى جودة البيانات وفق دليل سكرتارية الامم المتحدة لدقة العمر والنوع 


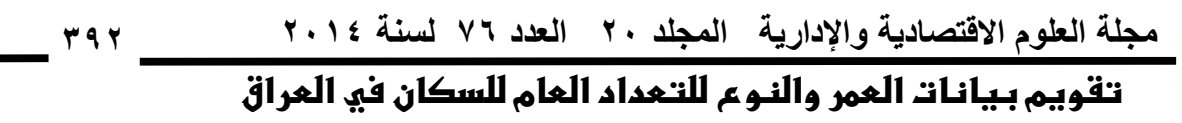

باستعمال مقدرات Kernel Bayesian اللإمعلمية

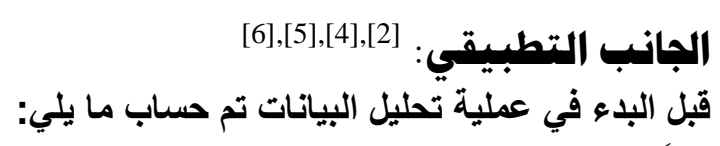

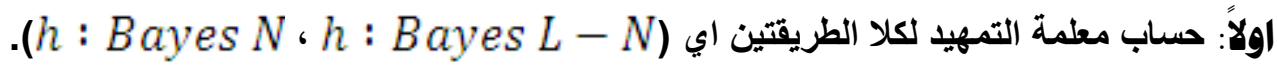

ثانيًا: حساب مقر (Nadaraya Watson Kernel) وحسب المعادلة التالية:

$\widehat{P}_{i}=\frac{\sum_{j=1}^{m} K\left(\frac{i / m-j / m}{h}\right) \bar{P}_{j}}{\sum_{j=1}^{m} K\left(\frac{i / m-j / m}{h}\right)}$

m تمثل عدد الفئات العمرية.

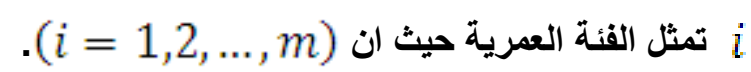

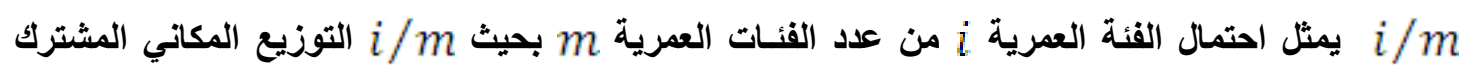

$\bar{P}_{i}$ (co-spatial design)

$\bar{P}_{i}=\frac{n i}{n}$

وان:

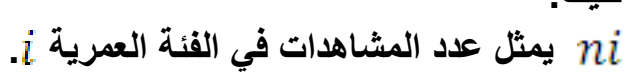
n يمثل العدد الكلي للمشاهدات.

يمثل التكرار النسبي لعدد المشاهدات بالفئة العمرية يمثل مقدر (Nadaraya Watson Kernel) بعد تحويل البيانات الى احتمال. Kernel تمثل دالة h

ووفق المعادلة (39) تم تحويل البيانات الى احتمال وبالتالي اصبحت مستمرة. إذ تم اعادة التمهيد لكل خلية اي لكل فئة عمرية. ثالثًا: تم مقارنة البيانـات قبل التمهيد مع البيانـات الناتجـة بعد استعمال طريقة Kernel التلامعلمية بواسطة

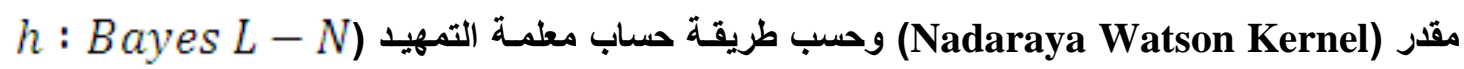
و عن ط:Bayes N ، 
باستعمال مقدرات Kernel Bayesian اللإمعلمية

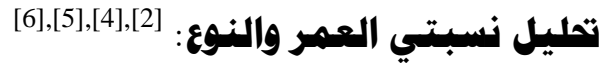

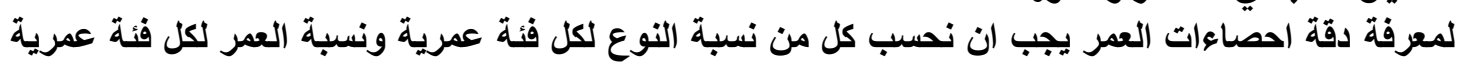

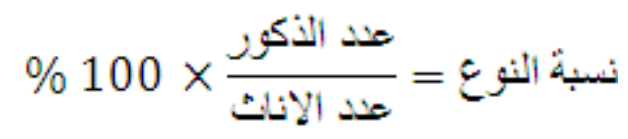

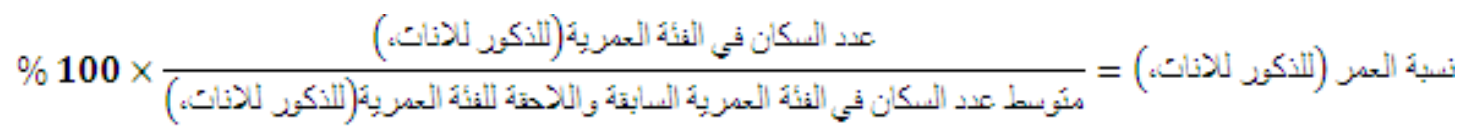
(42) ...

ومن هاتين النسبتين تم الحكم على مدى صحة البيانات الاصلية قبل التمهيد والبيانات التي تم التوصل اليها بعد استعمال طرائق التمهيا وبالتالي الكثف عن الاخطاء التي تقع في اعداد بعض التئ الفئات إذ عملية التحليل

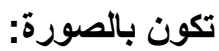

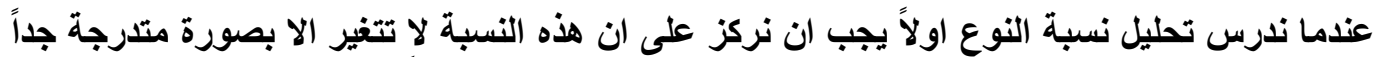

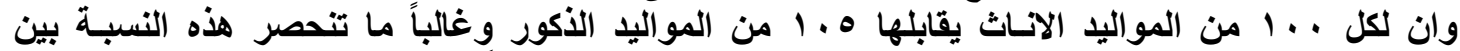

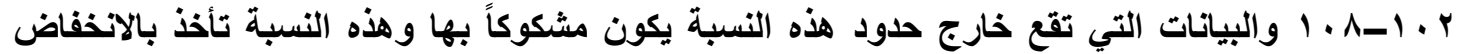

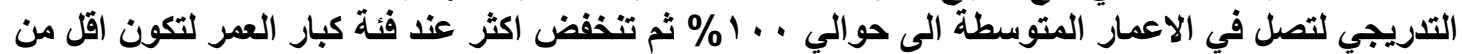

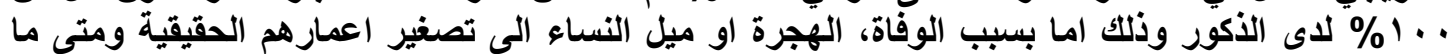

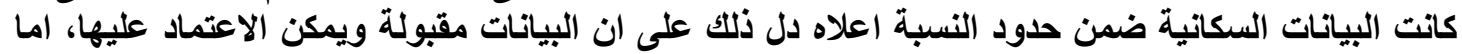

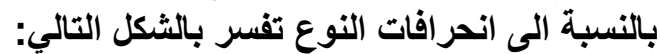

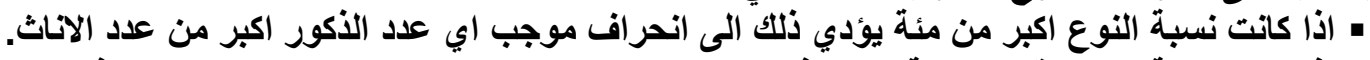

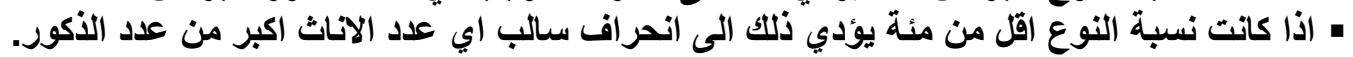

لتحليل نسبة العمر في المعادلة (ب ؛) يتم تقسيم الفئات العمرية الى ثلاث فئات عمرية عريضة وعلى النحو

• التحليل نسبل نسبة العمر في المعر:

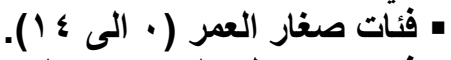

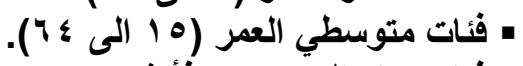

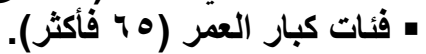

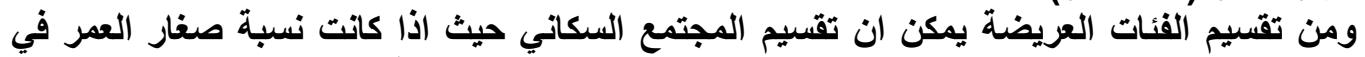

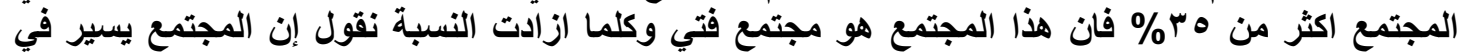

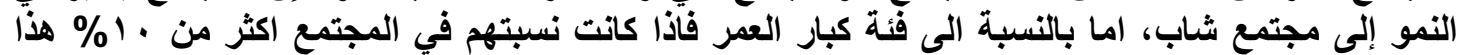

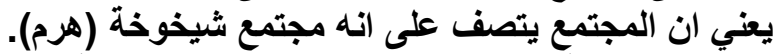

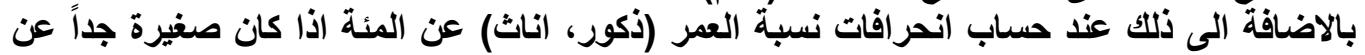

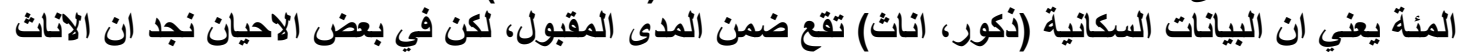

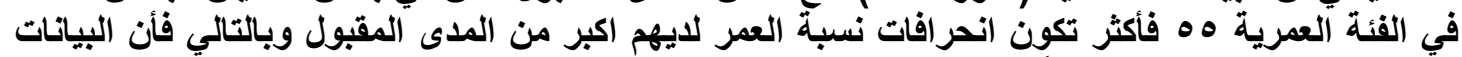

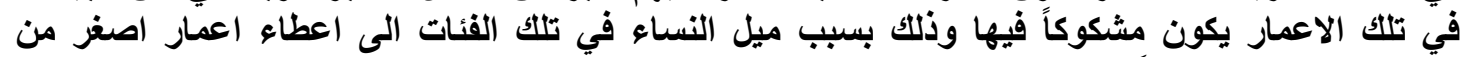

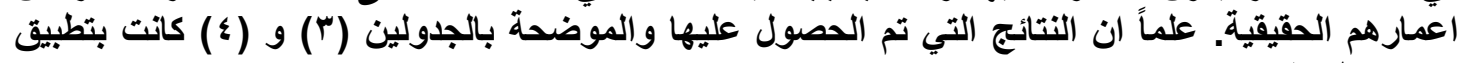
برنامج الماتلاب. 
باستعمال مقدرات Kernel Bayesian الإمعلمية

\begin{tabular}{|c|c|c|c|}
\hline h : Bayes N & h : Bayes L-N & البيانات قبل التمهي & فائات \\
\hline $1,905,693$ & $1,877,613$ & $1,911,828$ & $0-4$ \\
\hline $1,686,688$ & $1,661,835$ & $1,692,118$ & $5-9$ \\
\hline $1,439,919$ & $1,407,614$ & $1,425,042$ & $10-14$ \\
\hline $1,268,862$ & $1,227,083$ & $1,293,435$ & 15-19 \\
\hline $1,030,977$ & $1,034,103$ & $1,019,269$ & $20-24$ \\
\hline 846,857 & 844,418 & 849,385 & $25-29$ \\
\hline 664,684 & 670,975 & 680,816 & 30-34 \\
\hline 453,346 & 526,730 & 413,916 & $35-39$ \\
\hline 411,360 & 417,730 & 429,425 & $40-44$ \\
\hline 331,844 & 336,024 & 327,438 & $45-49$ \\
\hline 265,168 & 269,047 & 263,859 & $50-54$ \\
\hline 209,911 & 210,820 & 215,068 & $55-59$ \\
\hline 144,332 & 161,572 & 134,845 & $60-64$ \\
\hline 118,772 & 122,964 & 123,427 & $65-69$ \\
\hline 83,871 & 95,597 & 82,011 & 70-74 \\
\hline 54,937 & 54,128 & 55,114 & 75-79 \\
\hline 70,028 & 68,996 & 70,254 & $80+$ \\
\hline $10,987,249$ & $10,987,249$ & $10,987,249$ & لمجموع \\
\hline
\end{tabular}

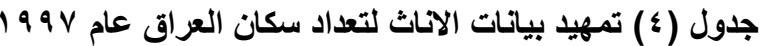

\begin{tabular}{|c|c|c|c|}
\hline h : Bayes N & h : Bayes L-N & البياتات قبل التمهيد & فئات العمر \\
\hline $1,861,583$ & $1,835,427$ & $1,867,103$ & $0-4$ \\
\hline $1,626,495$ & $1,603,642$ & $1,631,318$ & $5-9$ \\
\hline $1,383,646$ & $1,367,208$ & $1,366,867$ & $10-14$ \\
\hline $1,229,064$ & $1,202,127$ & $1,246,767$ & 15-19 \\
\hline $1,034,736$ & $1,030,261$ & $1,028,478$ & $20-24$ \\
\hline 870,848 & 860,999 & 875,086 & $25-29$ \\
\hline 699,795 & 701,691 & 710,286 & $30-34$ \\
\hline 512,610 & 561,969 & 486,257 & 35-39 \\
\hline 442,946 & 447,686 & 454,607 & $40-44$ \\
\hline 350,772 & 355,542 & 350,292 & $45-49$ \\
\hline 262,580 & 280,224 & 256,622 & $50-54$ \\
\hline 207,910 & 220,425 & 209,141 & $55-59$ \\
\hline 162,431 & 175,262 & 156,724 & $60-64$ \\
\hline 142,834 & 141,521 & 147,040 & 65-69 \\
\hline 109,916 & 116,441 & 111,100 & $70-74$ \\
\hline 68,750 & 67,784 & 68,954 & 75-79 \\
\hline 92,075 & 90,781 & 92,348 & $80+$ \\
\hline $11,058,992$ & $11,058,992$ & $11,058,992$ & المجموع \\
\hline
\end{tabular}

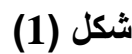

بيانـات الذكور قبل ويعد التمهيذ 


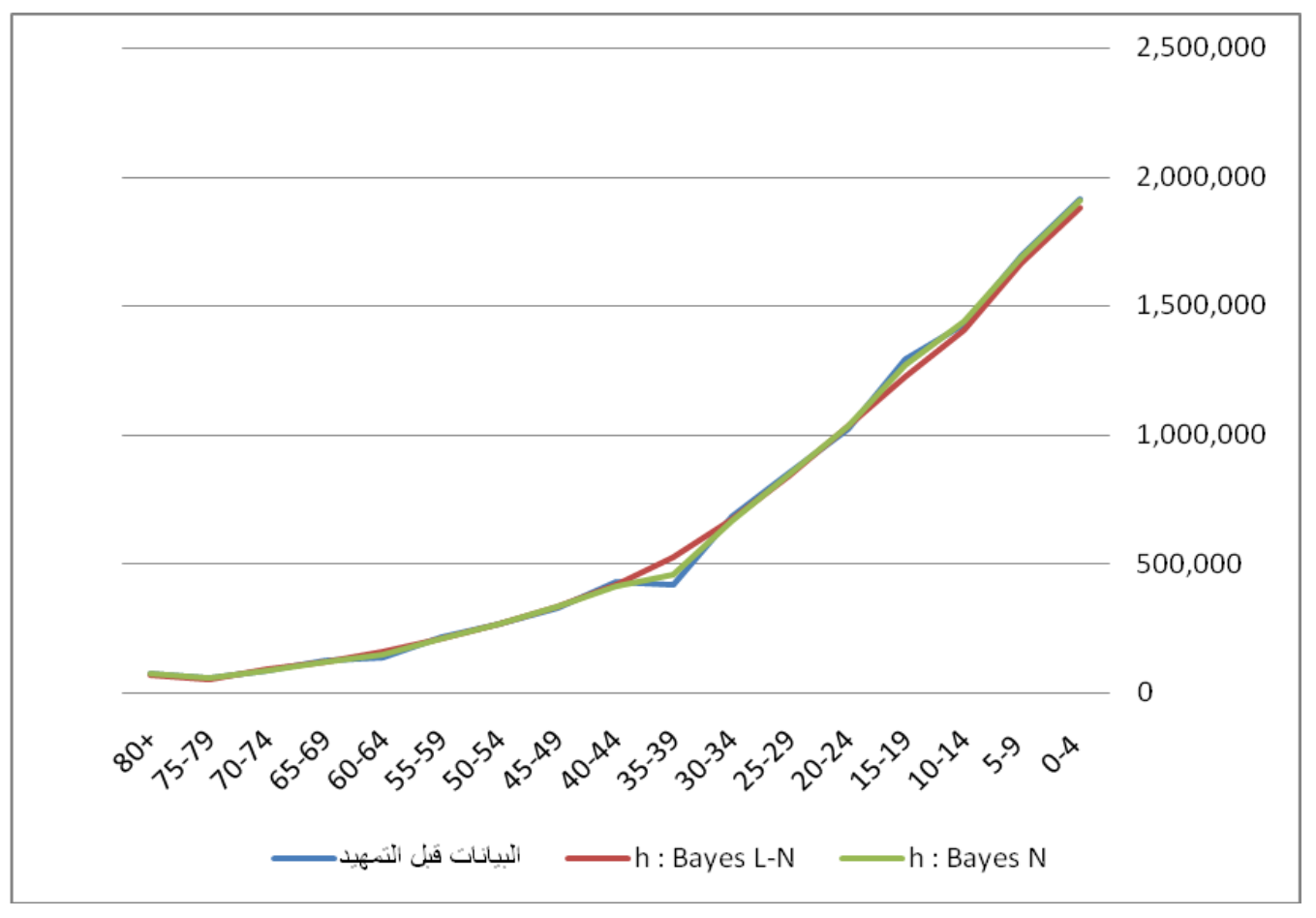

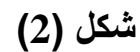

بيانات الاناث قبل وبعد التمهيد

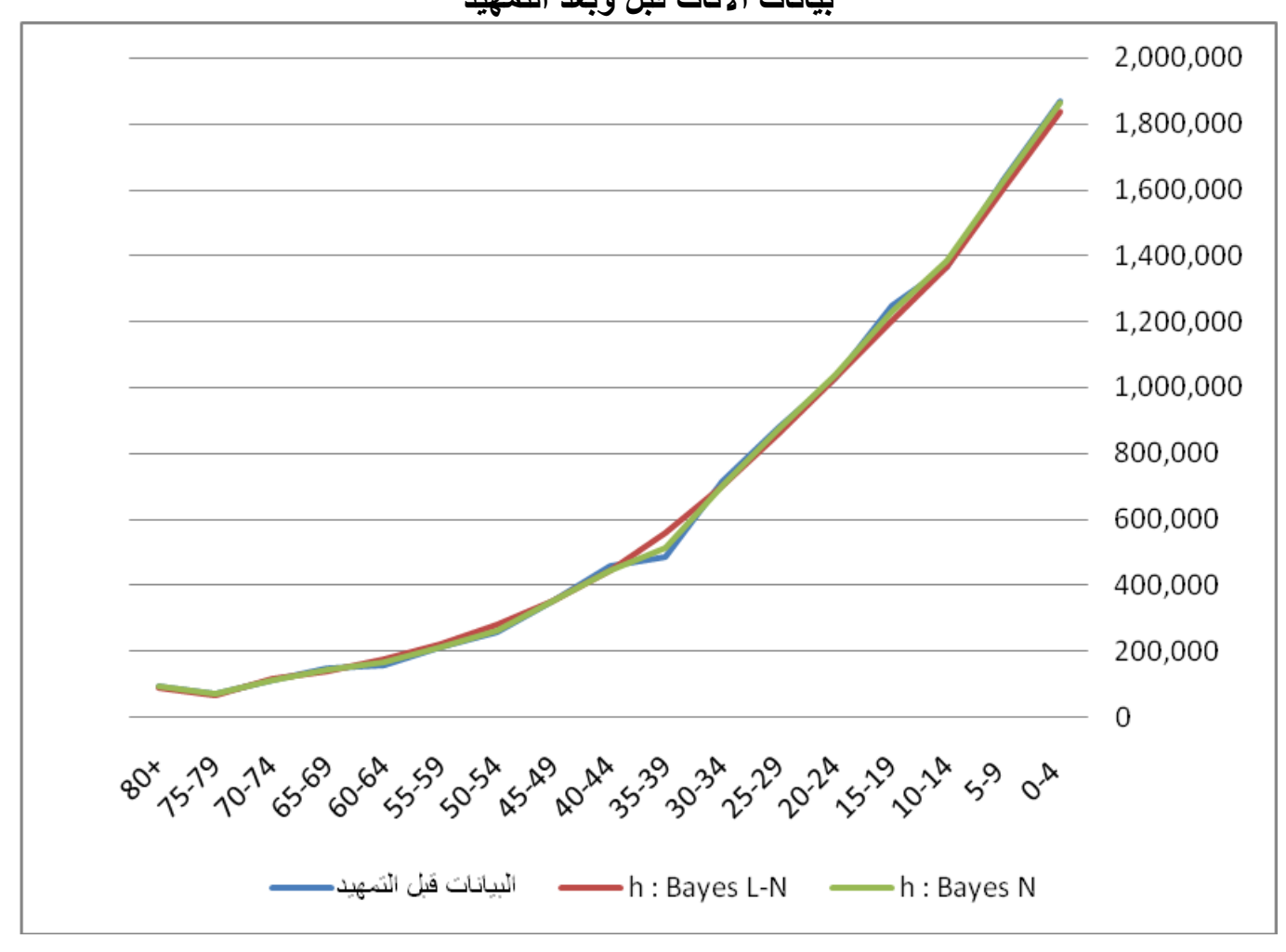




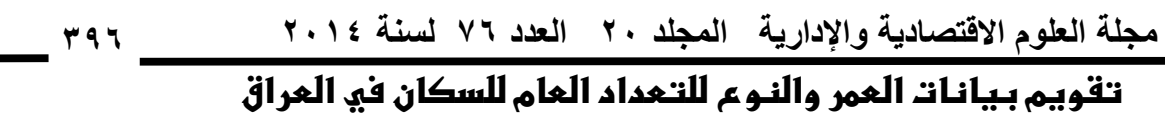

باستعمال مقدرات Kernel Bayesian الإمعلمية

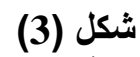

انحرافات الذكور عن المئة

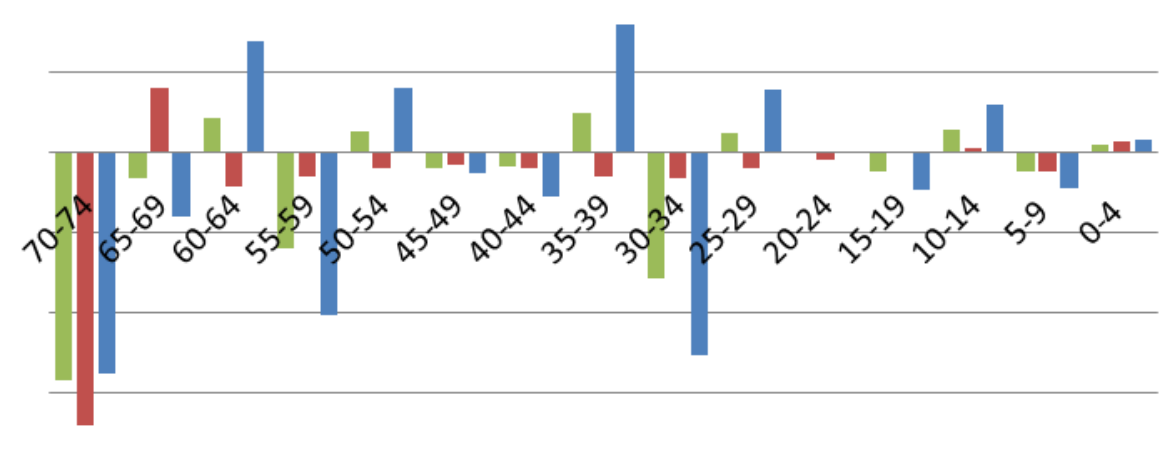

(4) شكل (1) (1)

انحرافات الاناث عن المئة

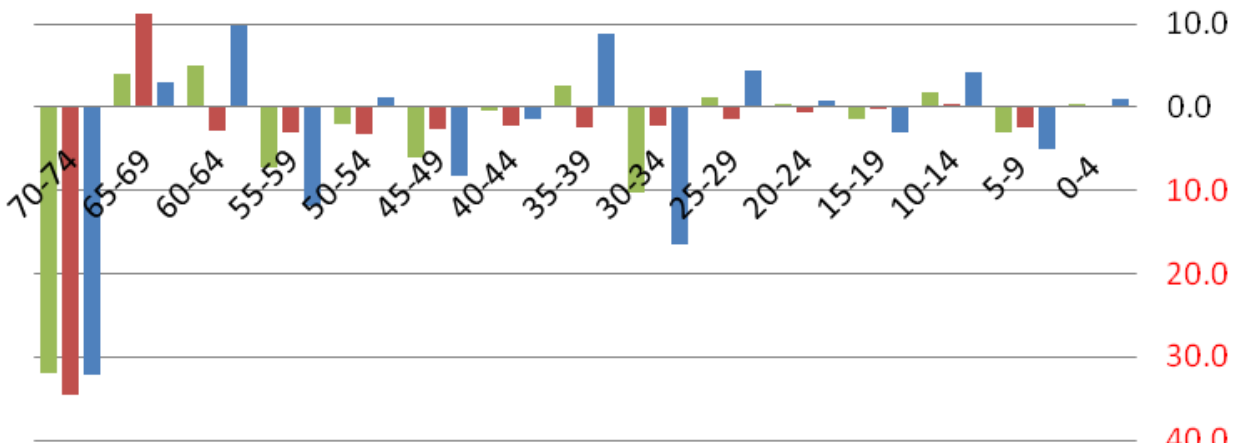




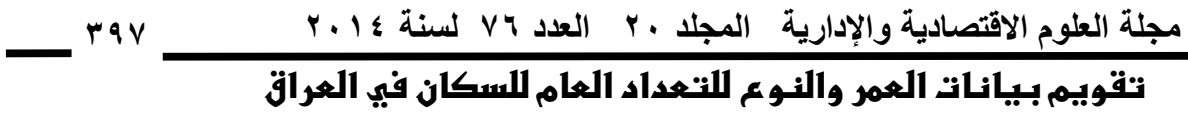

باستتعهال مقدرات Kernel Bayesian الإمعلمية

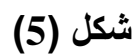

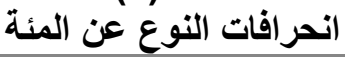

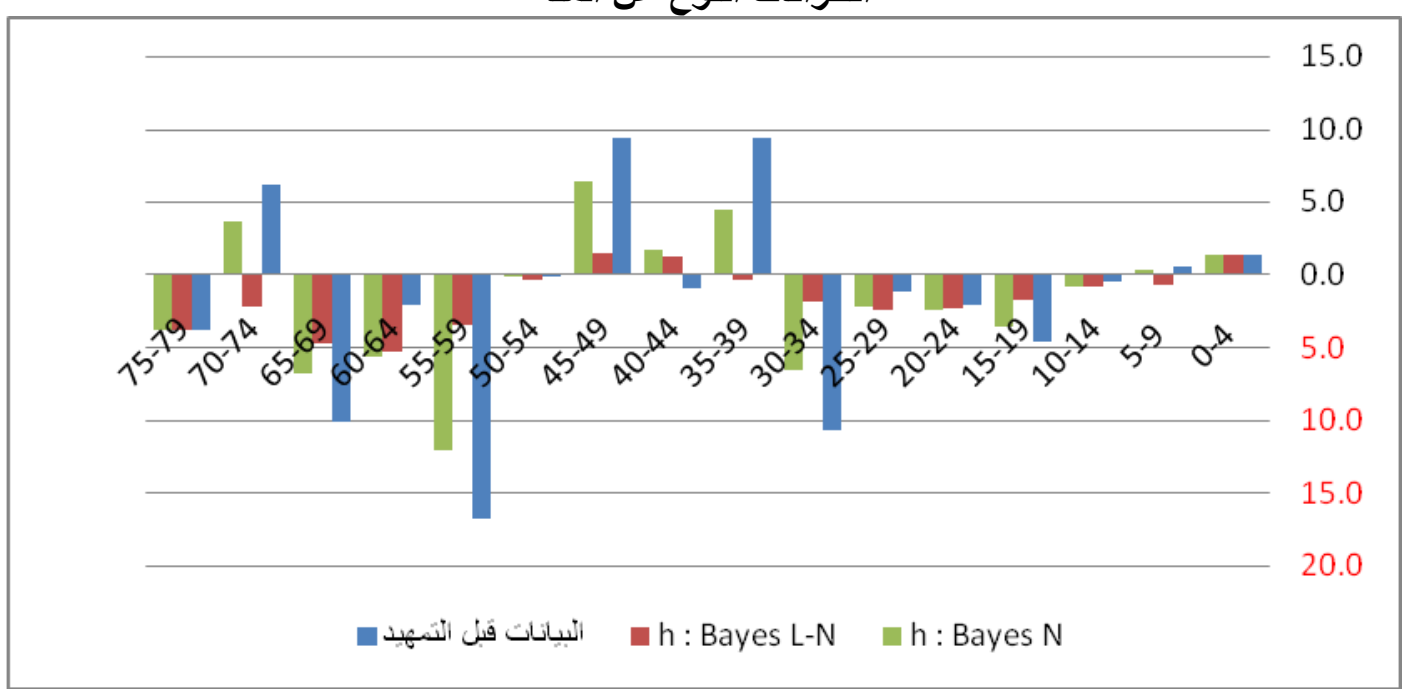

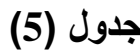

تحليل لنسبة العمر وقيمة دليل سكرتارية الامم المتحدة لدقة العمر والنوع للبيانات الاصلية والبيانات الناتجة

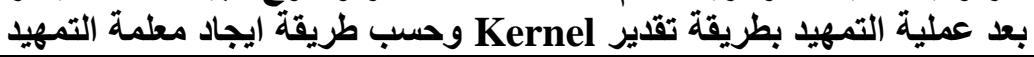

\begin{tabular}{|c|c|c|c|}
\hline h : Bayes N & h : Bayes L-N & Real data & فئات العمر \\
\hline $3,767,276$ & $3,713,040$ & $3,778,931$ & $0-4$ \\
\hline $3,313,183$ & $3,265,477$ & $3,323,436$ & $5-9$ \\
\hline $2,823,565$ & $2,774,822$ & $2,791,909$ & $10-14$ \\
\hline $9,904,024$ & $9,753,339$ & $9,894,276$ & مجموع فَئة صغار العمر \\
\hline $22,046,241$ & $22,046,241$ & $22,046,241$ & المجموع الكلي \\
\hline 44.9239 & 44.2404 & 44.8797 & معدل فئة صغار العمر \\
\hline
\end{tabular}

\begin{tabular}{|c|c|c|c|}
\hline h : Bayes N & h : Bayes L-N & Real data & فئات العمر \\
\hline 261,606 & 264,485 & 270,467 & 65-69 \\
\hline 193,787 & 212,038 & 193,111 & $70-74$ \\
\hline 123,687 & $\mathbf{1 2 1 , 9 1 2}$ & 124,068 & 75-79 \\
\hline 162,103 & 159,777 & 162,602 & $80+$ \\
\hline 741,183 & 758,212 & 750,248 & مجموع فئة كبار العمر \\
\hline $22,046,241$ & $22,046,241$ & $22,046,241$ & المجموع الكلي \\
\hline 3.3619 & 3.4392 & 3.4031 & معدل فئة كبار العمر \\
\hline
\end{tabular}

\begin{tabular}{|c|c|c|c|}
\hline 22.57 & 15.39 & 32.69 & دليل سكرتارية الامم المتحدة لاقة العمر \\
\hline
\end{tabular}




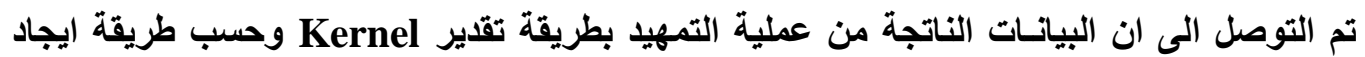

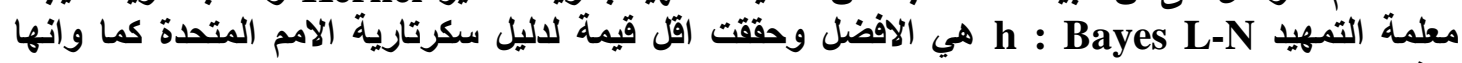
حافظت على نسبتي العمر والنوع.

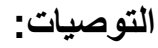

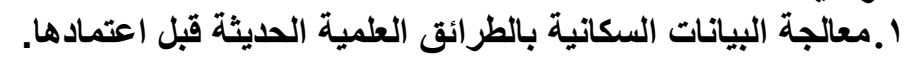

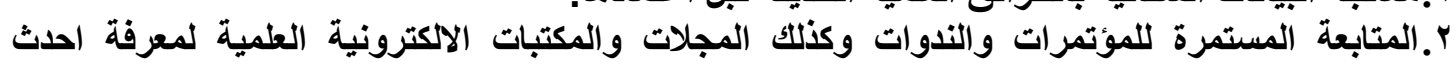

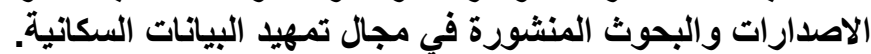

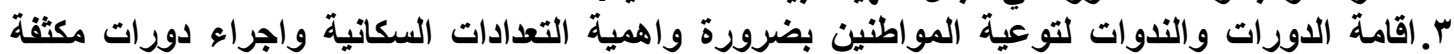
للكادر المكلف بعملية التعداد تجنباً لدوث لتون الاخطاء خصوصاً بالأطراف (الارياف).

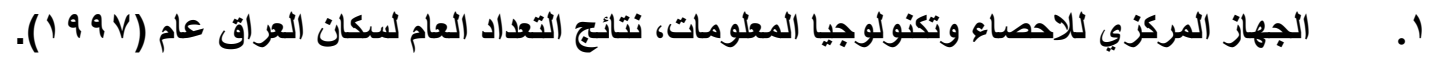

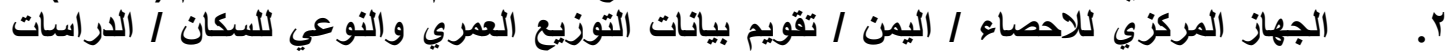

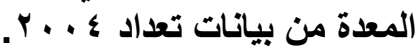

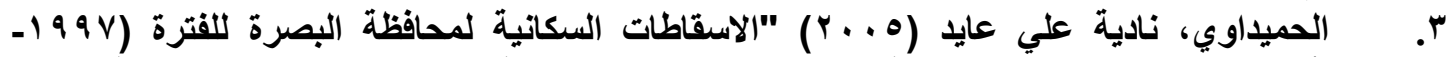

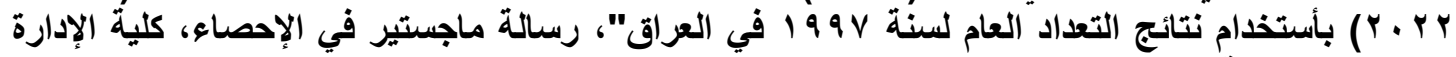

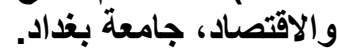

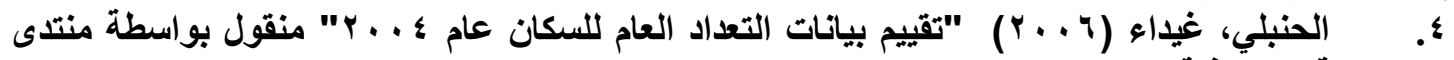

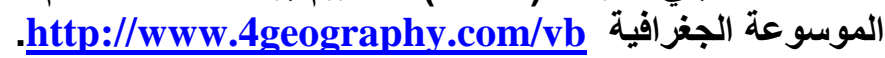
5.Arriaga, E. E., Johnson, P. D., Jamison, E. (1994) "Population analysis with microcomputers" Presentation of techniques, Vol. I.

6. Cula, S. , HOŞGÖR, S. (2006) "APPLICATION OF KERNEL ESTIMATION METHOD FOR CORRECTION OF AGE DISTRIBUTION ERRORS IN CENSUS" NüfusbilimDergisilTurkish Journal of Population Studies, pp. 61-71.

7. Hansen, B. E. (2009) "Lecture Notes on Nonparametrics" University of Wisconsin.

8. Kuruwita, C. (2006) "A BAYESIAN APPROACH FOR BANDWIDTH SELECTION IN KERNEL DENSITY ESTIMATION WITH CENSORED DATA" A Master's Thesis.

9. Stern, H. S. ,Gelman, A. , Carlin, J. B. , Rubin, D. B. (2003) "Bayesian Data Analysis" CHAPMAN \& HALL / CRC, Texts in Statistical Science Series.

10. Turlach, B. A. (1993) "Bandwidth Selection in Kernel Density Estimation: A Review" C.O.R.E. and Institut de Statistique. Universit e Catholique de Louvain.

11. Wikipedia, the free encyclopedia, "Kernel regression, Kernel density estimation and Kernel (statistics)".

12. Zucchini, W. (2003) "APPLIED SMOOTHING TECHNIQUES Part 1: Kernel Density Estimation" http://isc.temple.edu/economics /Econ616/Kernel/ast_part1.pdf .

13. Zheng, Q. (2009) "Local Adaptive Smoothing in Kernel Regression Estimation" A Master's Thesis.

Evaluation Age and Gender for General Census of the population in Iraq by using nonparametric Bayesian Kernel Estimators 


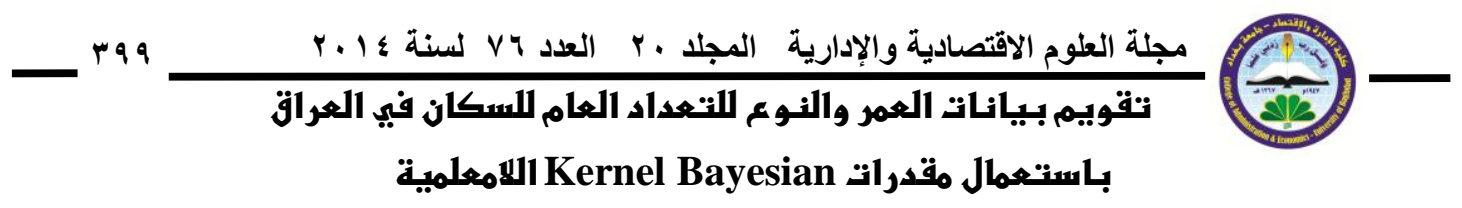

\begin{abstract}
The process of evaluating data (age and the gender structure) is one of the important factors that help any country to draw plans and programs for the future. Discussed the errors in population data for the census of Iraqi population of 1997. targeted correct and revised to serve the purposes of planning. which will be smoothing the population databy using nonparametric regression estimator (Nadaraya-Watson estimator) This estimator depends on bandwidth (h) which can be calculate it by two ways of using Bayesian method, the first when observations distribution is Lognormal Kernel and the second is when observations distribution is Normal Kernel.

then we will be compare between the result of these methods by using UN AgeSex Accuracy Index and analysis of the Age and Gender ratios to find the method which gave the optimum smoothing for data. And we reached that the method of estimate $h$ when observations distributed as Lognormal Kernel of Bayesian method is the best because it achieved less value of UN Age-Sex Accuracy Index.
\end{abstract}

keyword: Nadaraya-Watson Kernel estimator, inverted gamma prior distribution, Lognormal Kernel posterior distribution, Normal Kernel posterior distribution, UN Age-Sex Accuracy Index. 